\title{
INCIDENTES DE MAMÍFEROS NA RODOVIA BR-277, PARANÁ - BRASIL
}

\author{
LIST OF MAMMALS RUN-OVER IN \\ BR-277 HIGHWAY, PARANÁ STATE, BRAZIL
}

\author{
Mário Belão ${ }^{1}$, Roberto Bóçon ${ }^{2}$, Susete Wambier Christo ${ }^{3}$, Maria Antonia Michels de \\ Souza $^{4}$, João Luiz de Souza Júnior ${ }^{5}$ \\ ${ }^{1}$ Concessionária Ecovia Caminho do Mar S/A. E-mail: mario.belao@ecovia.com.br \\ ${ }^{2}$ Mater Natura Instituto de Estudos Ambientais. E-mail: rbocob@gmail.com \\ ${ }^{3}$ Universidade Estadual de Ponta Grossa, Departamento de Biologia Geral. \\ E-mail: swchristo@hotmail.com \\ ${ }^{4}$ Universidade Estadual de Ponta Grossa, Departamento de Biologia Geral. \\ E-mail: lagunense@hotmail.com \\ ${ }^{5}$ Graduando em Bacharelado em Ciências Biológicas - UEPG. E-mail: joao.ljr@gmail.com
}

Data de recebimento: 05/05/2014

Data da aprovação: 19/05/2014

\section{RESUMO}

Impactos causados nas rodovias sobre os grupos de vertebrados podem influenciar desde a alteração em seu habitat até sua morte. Portanto, o presente trabalho teve como objetivo avaliar a incidência de mamíferos atropelados na rodovia BR-277/PR e propor medidas mitigadoras para amenizar tais impactos. $\mathrm{O}$ estudo foi realizado entre maio de 2009 e dezembro de 2010 na rodovia BR-277 sob concessão da empresa Ecovia Caminho do Mar S/A nos sentidos Curitiba - Paranaguá - Curitiba. As amostragens foram realizadas semanalmente, com veículo operacional a uma velocidade média de $50 \mathrm{~km} / \mathrm{h}$. Os resultados obtidos mostraram 105 mamíferos silvestres atropelados distribuídos em 22 espécies pertencentes a seis ordens. As espécies mais vitimadas foram aquelas pertencentes à ordem Carnivora-Cerdocyon thous e Procyon cancrivorus, com atenção a Leopardus tigrinus e Lutra longicaudis, considerados vulneráveis no Estado do Paraná. As demais ordens mais atingidas foram Rodentia, Xenarthra, Marsupialia, Lagomorpha e Primate, sendo constatada a maior frequência de atropelamentos nos trechos entre os kilômetros 45 e 75 da rodovia.

Palavras-chave: Mata Atlântica. Impacto. Biodiversidade. Animais silvestres.

\begin{abstract}
Impacts generated by the construction of roads and highways over some vertebrate groups may trigger alterations especifically in their habitat and even in death by running over. The purpose of the present paper is to assess the incidence of mammals hit by vehicles in the highway BR-277 (Paraná State) and to present measures to mitigate this impacts. This study was conducted between May 2009 and December 2010 in the stretch of BR-277 under the concession of Ecovia - Caminho do Mar S/A in both directions between Curitiba and Paranaguá. Samplings were weekly performed with an operational vehicle at $50 \mathrm{~km} / \mathrm{h}$ average. Results yielded 105 wild mammals hit, comprising 22 species belonging to six orders. The most victimized species belong
\end{abstract}


to the order Carnivora (Cerdocyon thous and Procyon cancrivorus), with additional remarks to Felis tigrinus and Lutra longicaudis, both endangered species in the country. Other vitimized mammals belong to Rodentia, Xenarthra, Marsupialia, Lagomorpha and Primates. The highest rate of running over observed was between kilometers 45 and 75 .

Keywords: Atlantic Forest. Impact. Biodiversity. Wild animals.

\section{INTRODUÇÃO}

A ocorrência envolvendo animais os silvestres em rodovias é um fator a ser investigado em função dos efeitos que podem ocorrer na fauna local. Estudos realizados sobre a presença de animais em rodovias sugerem que fatores como: o tipo de formação vegetal; as condições climáticas; comportamento e abundância dos animais, especialmente os mamíferos de grande e médio porte que se deslocam às margens de rodovias preferencialmente à noite; bem como o lixo "jogado" pelos motoristas, possam contribuir para um maior índice de ocorrência com animais (PINOWSKI, 2005; MIRANDA et al., 2009).

Segundo GRIESE (2007), há ainda outros fatores diretamente ligados às rodovias e ao ambiente do entorno que contribuem para o atropelamento de fauna, como: tipo e grau de conservação da paisagem, largura e número de pistas, velocidade e intensidade de tráfego da rodovia, período do ano e abundância dos animais. Ainda há a capacidade de forrageamento ao longo do curso das rodovias, por meio de sementes e frutos oriundos de plantas que margeiam as pistas e animais mortos que atraem os carniceiros, ocasionando atropelamentos (MIRANDA et al., 2009).

Os animais mais suscetíveis são aqueles que não se adaptam a ambientes antropizados, os que possuem populações reduzidas e os que buscam o asfalto para se aquecer (SCHONEWALD-COX; BUECHNER, 1992).

Dentre os vários grupos de vertebrados, os mamíferos carnívoros podem ser considerados os mais suscetíveis à ação de atropelamentos. Segundo MELO; SANTOS-FILHO (2007), a frequência de ocorrência pode alterar sazonalmente, aumentando durante a época reprodutiva e/ou do recrutamento de jovens e filhotes.

A concessionária Ecovia - Caminho do Mar apresenta um Plano de Gestão Ambiental, especialmente ao longo da BR-277. No entanto são escassos os trabalhos descrevendo a distribuição de ocorrência envolvendo mamífero na rodovia BR-277 (Curitiba -
Paranaguá - Curitiba), informações estas importantes para elaboração de projetos de mitigação da problemática. Para subsidiar esse Plano, o presente trabalho teve como objetivo avaliar a incidência de mamíferos na rodovia BR-277/PR.

\section{MATERIAL E MÉTODOS}

O presente estudo foi desenvolvido na rodovia BR-277, trecho entre Curitiba - Paranaguá - Curitiba sob administração da empresa Ecovia - Caminho do Mar S/A, localizada no litoral sul do estado do Paraná, entre maio de 2009 e dezembro de 2010.

As amostragens foram realizadas semanalmente, com veículo operacional a uma velocidade média de $50 \mathrm{~km} / \mathrm{h}$, por meio de dois observadores. Em cada amostragem, a rodovia foi percorrida integralmente com deslocamento e coleta de informações nos dois sentidos: Curitiba - Paranaguá - Curitiba. Os animais encontrados foram fotografados e identificados, acondicionados em sacos plásticos, etiquetados e depositados no Museu de História Natural do Capão da Imbuia, em Curitiba - PR.

\section{Análise estatística}

Foi realizado um teste $t$ de Student para avaliar diferença da ocorrência de incidente com mamíferos (vd-variável dependente) entre os anos estudados (vi-variável independente). Uma análise de variância (ANOVA) unifatorial foi realizada para identificar diferença entre os trechos (vi) da rodovia (Classes: $0-15$; 15-30;30-45; 45-60; 60-75; 75-90 Km) e os incidentes com mamíferos (vd). Para ambas as análises foi avaliado se os dados eram paramétricos (teste de normalidade Shapiro-Wilk e homocedaticidade com o teste de Levene) e $\alpha=0,05$.

\section{RESULTADOS}

Os resultados obtidos entre maio de 2009 e dezembro de 2010 mostraram 105 mamíferos silvestres distribuídos em 22 espécies pertencentes 
a seis ordens. As espécies mais vitimadas foram aquelas pertencentes à ordem Carnivora Cerdocyon thous e Procyon cancrivorus, com atenção a Leopardus tigrinus e Lutra longicaudis, considerados vulneráveis no Estado do Paraná.

As demais ordens mais atingidas foram Rodentia (Coendou vilosus), Xenarthra (Tamandua tetradactyla), Marsupialia (Didelphis albiventris), Lagomorpha (Lepus europaeus) e Primate (Alouatta caraya) (Tabela 1).
Quando analisados os registros de ocorrência, observou-se uma predominância de incidentes com carnívoros e roedores nos anos estudados nos trechos entre os kilômetros 30-45 (9,5 \pm 4,95 animais), 45-60 (11,5 $\pm 3,53$ animais) e 60-75 (17,0 \pm 4,24 animais) (Tabelas 2 e 3; Figura 1), sem diferenças estatísticas ( $\mathrm{F}=3,91$; $\mathrm{p}=0,06)$. Nos dois anos as médias das ocorrências foram de 10,67 ( $\pm 6,50)$ em 2009 e 6,83 ( $\pm 4,07)$ em 2010, não diferindo entre os anos estudados $(t=1,22 ; p=0,25)$.

Tabela 1- Lista dos mamíferos atropelados na rodovia BR-277 administrada pela concessionária Ecovia - Caminho do Mar S/A durante 2009 e 2010. $N$ : número absoluto de indivíduos

\begin{tabular}{|c|c|c|c|c|}
\hline ORDEM & ESPÉCIE & NOME COMUM & $\begin{array}{c}2009 \\
N\end{array}$ & $\begin{array}{c}2010 \\
N\end{array}$ \\
\hline \multicolumn{5}{|c|}{ CARNIVORA } \\
\hline & Cerdocyon thous & cachorro-do-mato & 11 & 07 \\
\hline & Leopardus tigrinus* & gato-do-mato & - & 04 \\
\hline & Leopardus pardalis & jaguatirica & - & 01 \\
\hline & Leopardus wiedi & gato-maracajá & - & 01 \\
\hline & Galictis cuja & furão & - & 01 \\
\hline & Lutra longicaudis & lontra & - & 01 \\
\hline & Procyon cancrivorus & mão-pelada & 04 & 05 \\
\hline \multicolumn{5}{|c|}{ LACOMORPHA } \\
\hline & Lepus europeaeus & lebre-europeia & 08 & 04 \\
\hline \multicolumn{5}{|c|}{ MARSUPIALIA } \\
\hline & Didelphis albiventris & gambá-de-orelha-branca & 01 & 07 \\
\hline & Didelphis marsupialis & gambá-de-orelha-preta & 03 & 01 \\
\hline & Didelphis sp. & gambá & 02 & - \\
\hline \multicolumn{5}{|l|}{ PRIMATE } \\
\hline & Alouatta caraya* & bugio & 01 & - \\
\hline \multicolumn{5}{|c|}{ RODENTIA } \\
\hline & Coendou villosus & ouriço-cacheiro & 10 & 03 \\
\hline & Coendou villosus & ouriço & 02 & - \\
\hline & Hydrochoerus hydrochaeris & capivara & 03 & 03 \\
\hline & Myocastor coypus & ratão-do-banhado & 02 & 01 \\
\hline \multicolumn{5}{|c|}{ XENARTHRA } \\
\hline & Dasypus hybridus & tatu-galinha & - & 02 \\
\hline & Dasypus novemcinctus & tatu-mulita & - & 03 \\
\hline & Dasypus sp. & tatu & 03 & 01 \\
\hline & Tamandua tetradactyla & tamanduá-mirim & 09 & 01 \\
\hline
\end{tabular}

* Brasil (Ameaçado) e Paraná (Vulnerável - VU)

Tabela 2- Frequência relativa de ocorrência das ordens de mamíferos, em 2009 e 2010 dividida por classe de kilômetro no trecho da BR 277

\begin{tabular}{|c|c|c|c|c|c|c|c|c|c|c|c|c|}
\hline \multirow{2}{*}{ Classes } & \multicolumn{2}{|c|}{ Carnivora } & \multicolumn{2}{|c|}{ Lagomorpha } & \multicolumn{2}{|c|}{ Marsupialia } & \multicolumn{2}{|c|}{ Primate } & \multicolumn{2}{|c|}{ Rodencia } & \multicolumn{2}{|c|}{ Xenarthra } \\
\hline & 2009 & 2010 & 2009 & 2010 & 2009 & 2010 & 2009 & 2010 & 2009 & 2010 & 2009 & 2010 \\
\hline $0-15$ & 3,39 & 2,17 & - & - & - & 4,35 & - & - & 1,69 & - & 3,39 & - \\
\hline $15-30$ & 5,08 & 6,52 & - & - & 5,08 & - & - & - & 1,69 & - & 5,08 & 2,17 \\
\hline $30-45$ & 10,17 & 10,87 & 5,08 & - & - & - & - & - & 1,69 & - & 1,69 & 2,17 \\
\hline $45-60$ & 5,08 & 13,04 & 6,78 & - & 3,39 & 6,52 & 1,69 & - & 22,03 & 4,35 & 0,00 & 6,52 \\
\hline $60-75$ & 1,69 & 8,70 & 1,69 & 6,52 & 1,69 & 2,17 & - & - & 1,69 & 8,70 & 6,78 & 4,35 \\
\hline $75-90$ & - & 2,17 & - & 2,17 & - & 4,35 & - & - & - & 2,17 & 3,39 & - \\
\hline TOTAL & 25,42 & 43,48 & 13,56 & 8,70 & 10,17 & 17,39 & 1,69 & - & 28,81 & 15.22 & 20,34 & 15.22 \\
\hline
\end{tabular}


Figura 1- Frequência relativa dos registros de mamíferos atropelados, por classe em kilômetro, entre os anos 2009 e 2010 na rodovia BR 277 (Curitiba - Paranaguá - Curitiba)

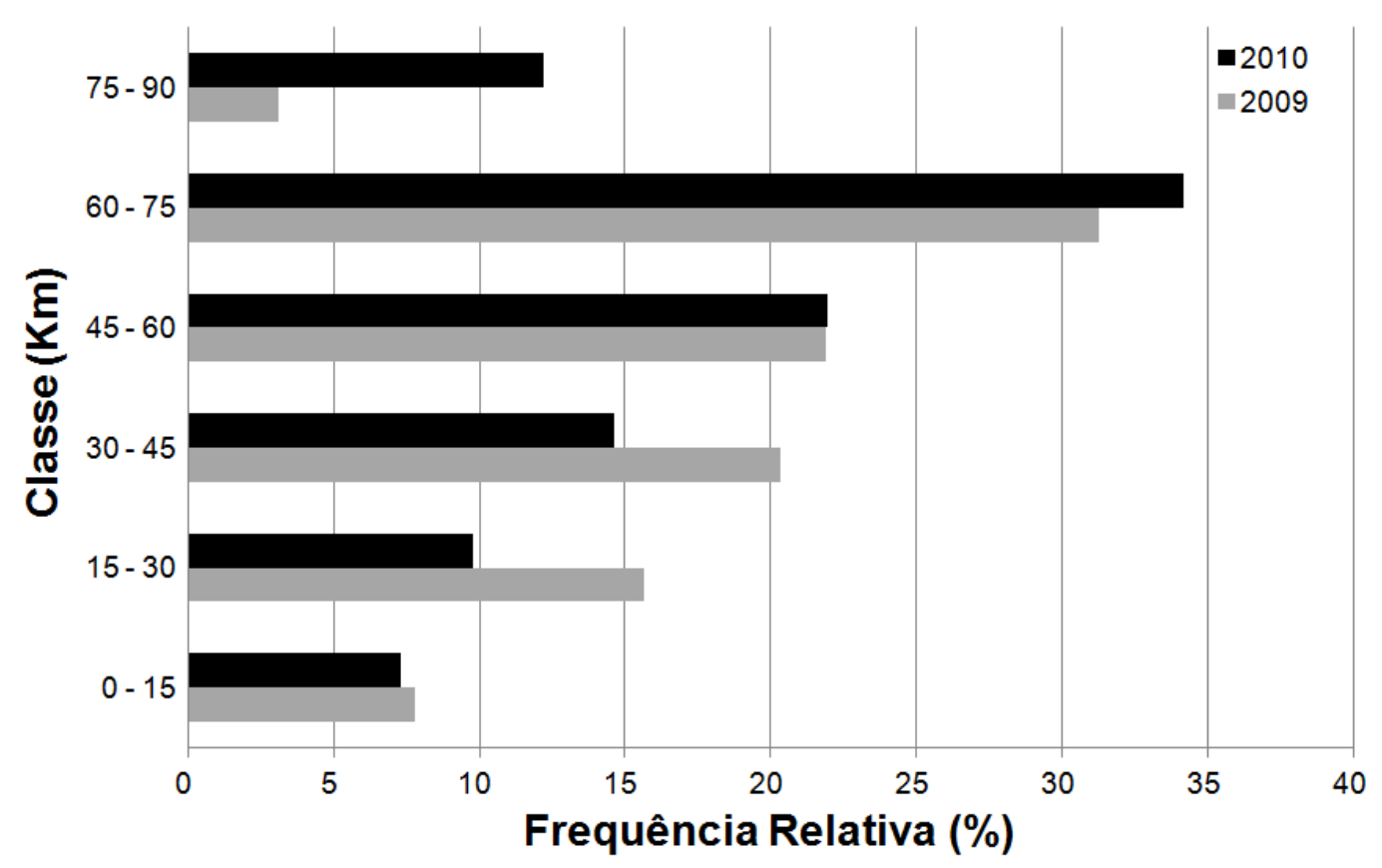

\section{DISCUSSÃO}

Nas rodovias, estaduais e/ou federais, a incidência de animais silvestres ocorre principalmente nas vias que contornam ou cortam áreas com vegetação densa e de campo, ou próximas de unidades de conservação. Várias espécies de vertebrados pertencentes às classes dos anfíbios, répteis, aves e mamíferos são vitimadas nas estradas brasileiras (BENCKE; BENCKE, 1999; PRADO et al., 2006; MELO; SANTOS-FILHO, 2007; SILVA et al., 2007; GUMER-COSTA; SPERBER, 2009; TURCI; BERNARDE, 2009).

Estudos realizados nas rodovias do norte do Brasil mostram uma frequência maior de anfíbios e répteis, seguido de aves e mamíferos (TURCI; BERNARDE, 2009; SPERBER; GUMIER-COSTA, 2009; PEREIRA et al., 2006).

No Estado de Goiás, na BR 153/GO-060, um estudo do impacto da rodovia próxima da unidade de conservação, o Parque Altamiro de Moura Pacheco, sobre os vertebrados encontrou um percentual elevado de indivíduos mortos por atropelamento das classes Mammalia, Aves, Repteis e Amphibia, em ordem decrescente (PRADO et al., 2006).
Enquanto no sul do país as maiores ocorrências abrangem as aves (NOVELLI et al., 1988) e principalmente os mamíferos, englobando, nesse último, as ordens Carnivora, Marsupialia e Xenartra como as mais vitimadas (CHEREM et al., 2007). No estado do Paraná, no trecho entre os quilômetros 30 e 75 da BR 277, os carnívoros Cerdocyon thous (cachorro-do-mato) e Procyon cancrivous (mãopelada) e xenartra $T$. tetradactyla (tamanduá-mirim) foram os mais frequentes. Estas espécies são comuns nos registros de ocorrências nas estradas brasileiras, especialmente $C$. thous encontrada por MELO; SANTOS-FILHO (2007) em áreas de borda de mata, e outros autores como PEREIRA et al. (2006) e CHEREM et al. (2007).

Algumas espécies de felinos, apesar de menos frequentes, como Leoparudus tigrinus (gato-do-mato), L. pardalis (jaguatirica) e L. wiedi (gato maracajá) merecem atenção, por encontrarem-se classificados como vulnerável para o Brasil e Paraná. Estas espécies foram encontradas entre os kilômetros 30 e 60 em áreas mistas com trechos de florestas preservadas, sendo tais espécies também observadas nas rodovias do Estado de Santa Catarina (CHEREM et al., 2007).

Assim como os felinos, os marsupiais Didelphis albiventris (gambá-de-orelha-branca) e Lontra 
longicaudis (lontra) foram raros na amostragem. $D$. albiventris foi frequente no trecho dos kilômetros 60-75, próximo à área urbana da estrada, local interessante para esta espécie que encontra com facilidade do alimento (rejeitos humanos). HENGEMÜHLE; CADEMARTORI (2008) e SPERBER; GUMIER-COSTA (2009) registraram a presença de gambás e lontra em condições semelhantes na Floresta Nacional de Carajás, Pará.

Dentre as ações mitigadoras efetuadas pela Concessionária da BR-277 podemos destacar os sonorizadores, placas de orientação ao usuário tanto na rodovia como nos SAUS e limpeza de resíduos no entorno da rodovia.

Os resultados do presente estudo podem auxiliar no Plano de Gestão Ambiental da Concessionária Ecovia ao longo da rodovia BR 277, possibilitando mais ações mitigadoras nos trechos de maior ocorrência envolvendo mamíferos.

\section{REFERÊNCIAS}

BENCKE, G. A.; BENCKE, C. S. C. The potential importance of road deaths as cause of mortality for large forest owls in southern Brazil. Cotinga, v.11, p.79-80, 1999.

CHEREM, J. J.; KAMMERS, M.; GHIZONI-JR, I. R.; MARTINS, A. Mamíferos de médio e grande porte atropelados em rodovias do Estado de Santa Catarina, sul dos Brasil. Biotemas, v.20, n.2, p.81-96, 2007.

GRIESE, J. Helmintofauna de vertebrados atropelados em rodovias da região de Botucatu, São Paulo. 2007. 68f. (Dissertação de Mestrado). Botucatu: Instituto de Biociências, Universidade Estadual Paulista.

HENGEMÜHLE, A.; CADEMARTORI, C. V. Levantamento de mortes de vertebrados silvestres devido a atropelamento em um trecho da estrada do mar (RS-389). Biodiversidade Pampeana, v.6, n.2, p.4-10, 2008.

MELO, E. S.; SANTOS-FILHO, M. Efeitos da BR-070 na Província Serrana de Cáceres, Mato Grosso, sobre a comunidade de vertebrados silvestres. Rev. Brasil. de Zoociências, v.9, n.2, p.185-192, 2007.

MIRANDA, C. M. S.; NASCIMENTO, A. A. T.; CARVALHO, G. D. Impacto do atropelamento de animais silvestres em trecho da BR 482, Minas Gerais BR. In: ENCONTRO INTERNACIONAL DE MEDICINA DA CONSERVAÇÃO, 2, UFRPE - Recife, PE, 2009.

NOVELLI, R.; TAKASE, E.; CASTRO, V. Estudo das aves mortas por atropelamento em um trecho da rodovia BR471, entre os distritos da Quinta e Taim, Rio Grande do Sul, Brasil. Rev. Brasil. de Zoologia, v.5. n.3, p.441-454, 1988.
PEREIRA, A. P. F. G.; ANDRADE, F. A. G.; FERNANDES, M. E. B. Dois anos de monitoramento dos atropelamentos de mamíferos na rodovia PA-458, Bragança, Pará. Bol. Mus. Para. Emílio Goeldi, Ciências Naturais, v.1, n.3, p.77-83, 2006.

PINOWSKI, J. Roadkills of vertebrates in Venezuela. Revista Brasileira de Zoologia v.22, n.1, p.191-196, 2005.

PRADO, T. R.; FERREIRA, A. A.; GUIMARÃES, Z. F. S. Efeito da implantação de rodovias no cerrado brasileiro sobre a fauna de vertebrados. Acta Sci. Biol. Sci. v.28. n.3, p.237-241, 2006

SCHONEWALD-COX, C.; BUECHNER, M. P. Protection and public roads. In: FIELDER, P. L.; JAIN, S. K. (Eds.). Conservation Biology: the theory and practice of nature conservation, preservation and management. London: Chapman and Hall, 1992. p. 375-395

SIlVA, M. O.; OLIVEIRA, I. S.; CARDOSO, M. W.; GRAF, V. Impacto dos atropelamentos sobre a herpetofauna da Floresta Atlântica (PR-340, Antonina, Paraná). Acta. Biol. Par., v.36, n.1-2, p.103-112, 2007.

SPERBER, C. F.; GUMIER-COSTA, F. Atropelamentos de vertebrados na Floresta Nacional de Carajás, Pará, Brasil. Acta Amazonica, v.39, n.2, p.459-466, 2009.

TURCI, L. C. B.; BERNARDE, P. S. Vertebrados atropelados na rodovia estadual 383 em Rondônia, Brasil. Biotemas, v.22, n.1, p.121-127, 2009. 